Original article

\title{
Features of cytokine signaling forming T-helper immune response in COPD of varying severity
}

\author{
Tatyana I. Vitkina, Karolina A. Sidletskaya
}

Vladivostok Branch of Far Eastern Scientific Center of Physiology and Pathology of Respiration - Institute of Medical Climatology and Rehabilitative Treatment, Vladivostok, Russia

Received 26 March 2019, Revised 22 November 2019, Accepted 27 April 2020

(C) 2019, Vitkina T.I., Sidletskaya K.A.

(C) 2019, Russian Open Medical Journal

Abstract: Introduction - Currently, chronic obstructive pulmonary disease (COPD) is a global public health problem. However, molecular mechanisms of the development of this pathology are still poorly understood. The aim is to establish mechanisms of cytokine regulation of T-helper (Th) immune pathway in patients with COPD of varying severity.

Material and Methods - The study included 112 patients with stable COPD (mild, moderate and severe grade) and 32 healthy volunteers (control group). We investigated serum cytokine levels (tumor necrosis factor- $\alpha$ (TNF- $\alpha$ ), interferon- $\gamma$ (IFN- $\gamma$ ), interleukin 4 (IL-4), IL-6, IL-10, IL-17A) and the percentage of circulating Th cells (CD4 ${ }^{+}$) expressing membrane receptor to IL-6 (IL-6R or CD126+), using flow cytometry. The levels of transforming growth factor- $\beta 1$ (TGF- $\beta 1$ ) and IL-21 were detected by ELISA. The direction of immune response in COPD patients was determined depending on the prevalence of cytokines playing a crucial role in the formation of certain Th cells type (Th1, Th17).

Results - Th1-associated cytokine profile was expressed at the initial stage of COPD; the Th17-associated cytokine profile begins to prevail at severe COPD. Among COPD patients with Th1-associated cytokine profile, a statistically significant increase in the number of $\mathrm{CD}^{+} \mathrm{CDD}^{+} \mathrm{C6}^{+}$ cells in comparison with the control group was identified only in severe COPD. In the group of COPD patients with Th17-associated cytokine profile, an increase in the number of $\mathrm{CD} 4{ }^{+} \mathrm{CD} 126^{+}$cells were observed at all severity stages of the pathology.

Conclusion - Moderate and severe COPD are characterized by the predominance of Th17-associated cytokine profile leading to chronic inflammation. The increase in IL-6R expression levels in circulating CD4+ cells serves as the mechanism for enhancing Th17-associated response in COPD.

Keywords: chronic obstructive pulmonary disease, T-helper immune response, cytokine signaling, interleukin-6 receptor.

Cite as Vitkina TI, Sidletskaya KA. Features of cytokine signaling forming T-helper immune response in COPD of varying severity. Russian Open Medical Journal 2020; 9: e0204.

Correspondence to Karolina A. Sidletskaya. Address: 690105, Russkaya Street 73-g, Vladivostok, Russia Federation. E-mail: d-karolina-a@mail.ru.

\section{Introduction}

Nowadays, chronic obstructive pulmonary disease (COPD) is a global medical and social problem. Professionals' concerns about COPD are due to the high risk of serious complications and rapid progression of pathology [1]. Currently, COPD is considered from the perspective of systemic inflammation. According to modern concepts, an important role in the regulation of this process belongs to various subpopulations of T-helper cells (type $1 \mathrm{~T}$ helper cells (Th1), Th2, Th17, T-regulatory cells (Treg), etc.) that determine the direction of the development of the immune response $[2,3]$. Studies focused on the definition of immunological phenotypes of COPD have controversial results. Some reports have shown that Th1 model of the immune response predominates in COPD patients, while other scientific groups have established Th2 phenotype in COPD [4]. An increase in the number of Th17 cells in the peripheral blood in COPD patients has been found in a number of studies [2, 3]. This fact indicates the important role of Th17 immune response in COPD pathophysiology.

The cytokine microenvironment creates the conditions for the deviation of Th immune response. Interferon- $\gamma$ (IFN- $\gamma$ ) is known to promote the differentiation of naive $\mathrm{CD}^{+}$lymphocytes into Th1 cells. Interleukin-4 (IL-4) is a key factor in the differentiation of Th2 cells. Cytokines such as IL-6, IL-17, IL-21 and transforming growth factor- $\beta 1$ (TGF- $\beta$ ) are involved in the formation of Th17 subpopulation and maintenance of the function of these cells [5].

IL-6 is one of the most important markers of systemic inflammation and the proposed therapeutic target for COPD [6]. In a recent study, Singh S. et al. have demonstrated the relationship between an increase in IL- 6 blood level and a decrease in lung function in COPD patients [7]. In addition, its role as a predictor of mortality in the disease has been shown [8]. According to modern concepts, the biolog cal effects of IL- 6 largely depend on signal pathway. There are two main types of IL- 6 signaling: classical and trans-signaling [9]. Data on IL-6 classical signaling at different stages of COPD are absent. Thus, the role of cytokine regulation disorders in the development of COPD is undeniable, however, more detail research on their mechanisms are required.

The aim of the study is to establish mechanisms of cytokine regulation of Th immune pathway in patients with COPD of varying severity. 
Table 1. Characteristics of study subjects

\begin{tabular}{|c|c|c|c|c|}
\hline Characteristics & Control group & Group 1 & Group 2 & Group 3 \\
\hline Total number & 32 & 36 & 52 & 24 \\
\hline Sex, male / female & $22 / 10$ & $25 / 11$ & $34 / 18$ & $13 / 11$ \\
\hline Age, years & $42.0 \pm 3.4$ & $55.4 \pm 2.1$ & $57.2 \pm 3.9$ & $60.6 \pm 2.3$ \\
\hline $\mathrm{BMI}, \mathrm{kg} / \mathrm{m}^{2}$ & $27.2 \pm 1.5$ & $20.4 \pm 3.9$ & $21.2 \pm 1.4$ & $20.9 \pm 3.8$ \\
\hline Smoking status, smokers / ex-smokers & 0 & $24 / 6$ & 35 / 11 & $16 / 7$ \\
\hline Smoking index, pack/year & 0 & $23.7 \pm 5.9$ & $29.3 \pm 4.7$ & $27.1 \pm 6.5$ \\
\hline Post-bronchodilator FEV1,\% of the normal & $104.8 \pm 2.1$ & $90.1 \pm 2.0$ & $73.9 \pm 2.6$ & $48.6 \pm 1.8$ \\
\hline CAT test, score & - & 4 & 9 & $<10$ \\
\hline
\end{tabular}

Data (age, BMI, smoking index and post-bronchodilator FEV1) are presented as mean with standard deviation - M \pm SD.

Group 1, patients with mild COPD; Group 2, patients with moderate COPD; Group 3, patients with severe COPD.

Table 2. Cytokine levels and percentage of $\mathrm{CD4}^{+} \mathrm{CD} 126^{+}$cells in the blood of healthy subjects and COPD patients with Th1-associated cytokine profile

\begin{tabular}{|c|c|c|c|c|}
\hline Parameters & Control group & Subgroup $1 a, n=24$ & Subgroup $2 a, n=24$ & Subgroup $3 a, n=6$ \\
\hline IL-4, pg/ml & $77.9(66.2,81.0)$ & $45.4(43.0,52.3)^{*}$ & $56.2(45.4,61.3)^{*}$ & $64.3(54.8,73.8)$ \\
\hline IL-6, pg/ml & $38.2(35.7,39.0)$ & $40.0(37.1,46.2)$ & $43.6(37.0,53.6)$ & $60.5(52.7,62.7)^{*}$ \\
\hline IL-10, pg/ml & $32.4(31.1,33.7)$ & $36.5(29.0,41.0)$ & $59.6(52.4,62.4)^{*}$ & $39.7(33.0,43.0)$ \\
\hline IL-21, pg/ml & $75.5(74.0,82.0)$ & $81.6(72.3,92.4)$ & $68.0(52.0,80.0)$ & $51.5(48.0,55.0)^{*}$ \\
\hline TNF- $\alpha, \mathrm{pg} / \mathrm{ml}$ & $46.3(43.2,48.9)$ & $93.0(89.6,107.5)^{*}$ & $59.9(52.4,62.4)^{*}$ & $146.7(145.7,147.8)^{*}$ \\
\hline IFN- $-\gamma, \mathrm{pg} / \mathrm{ml}$ & $103.5(91.6,125.7)$ & $325.3(308.1,344.0)^{*}$ & $155.2(125.9,187.3)^{*}$ & $195.6(191.5,209.6)^{*}$ \\
\hline TGF- $\beta 1, \mathrm{pg} / \mathrm{ml}$ & $150.0(118.0,180.0)$ & $162.0(88.0,184.0)$ & $158.5(145.0,205.0)$ & $215.0^{*}(210.0,220.0)^{*}$ \\
\hline $\mathrm{CD}^{+}{ }^{+} \mathrm{CD} 126^{+}$cells, $\%$ & $1.75(1.62,1.79)$ & $2.4(2.26,2.72)$ & $1.5(1.30,2.13)^{*}$ & $3.7(3.40,4.20)^{* * *}$ \\
\hline
\end{tabular}

Data are presented as median with lower and upper quartiles- Me (LQ, UQ). Statistical significance of differences in comparison with the control group according to Bonferroni correction: * $-p<0.00714 ; * * *-p<0.00014$.

Subgroup 1a, patients with mild COPD and Th1-associated cytokine profile; Subgroup 2a, patients with moderate COPD and Th1-associated cytokine profile; Subgroup 3a, patients with severe COPD and Th1-associated cytokine profile.

Table 3. Cytokine levels and percentage of CD4+CD126+ cells in the blood of healthy subjects and COPD patients with Th17-associated cytokine profile

\begin{tabular}{|c|c|c|c|c|}
\hline Parameters & Control group & Subgroup $1 b, n=12$ & Subgroup $2 b, n=28$ & Subgroup $3 b, n=12$ \\
\hline IL-4, pg/ml & $77.9(66.2,81.0)$ & $59.3(48.3,65.8)^{*}$ & $62.4(58.0,71.2)$ & $81.2(77.0,99.4)$ \\
\hline IL-6, pg/ml & $38.2(35.7,39.0)$ & $78.1(64.7,80.3)^{*}$ & $90.1(78.5,106.7)^{*}$ & $79.0(9.1,84.5)^{*}$ \\
\hline IL-10, pg/ml & $32.4(31.1,33.7)$ & $43.6(36.3,46.1)^{*}$ & $58.9(40.3,66.7)^{*}$ & $69.8(61.0,92.1)^{*}$ \\
\hline IL-21, pg/ml & $75.5(74.0,82.0)$ & $200.0(152.0,252.0)^{*}$ & $208.5(168.0,249.0)^{*}$ & $254.0(250.0,276.0)^{*}$ \\
\hline TNF- $\alpha, p g / m l$ & $46.3(43.2,48.9)$ & $59.4(49.0,69.0)^{*}$ & $55.7(42.5,62.8)$ & $70.2(60.0,94.0)^{*}$ \\
\hline IFN- $\nu, p g / m l$ & $103.5(91.6,125.7)$ & $125.9(114.0,151.0)$ & $110.2(107.5,138.0)$ & $108.0(102.0,161.1)$ \\
\hline TGF- $\beta 1, p g / m l$ & $150.0(118.0,180.0)$ & $180.0(175.0,275.0)^{*}$ & $204.5(195.0,275.0)^{*}$ & $280.0(240.0,340.0)^{*}$ \\
\hline $\mathrm{CD}^{+}{ }^{+} \mathrm{CD} 126^{+}$cells, $\%$ & $1.75(1.62,1.79)$ & $2.58(2.13,2.71)^{*}$ & $3.79(2.53,4.15)^{* *}$ & $6.75(5.50,8.50)^{* *}$ \\
\hline
\end{tabular}

Data are presented as median with lower and upper quartiles- Me (LQ, UQ). Statistical significance of differences in comparison with the control group according to Bonferroni correction: * $-p<0.00714 ;^{* *}-p<0.00143$.

Subgroup $1 \mathrm{~b}$, patients with mild COPD and Th17-associated cytokine profile; Subgroup 2b, patients with moderate COPD and Th71-associated cytokine profile; Subgroup $3 b$, patients with severe COPD and Th17-associated cytokine profile.

\section{Material and Methods}

\section{Study subjects}

The study included 112 patients with stable COPD who were divided into groups in accordance with disease severity: 36 people with mild COPD (group 1), 52 people with moderate COPD (group 2 ), and 24 people with severe COPD (group 3). Criteria for exclusion from the study were: acute infectious diseases, chronic diseases of internal organs in acute phase, and chronic heart failure in decompensation stage. The control group consisted of 32 practically healthy volunteers, non-smokers, with normal pulmonary function (Table 1).

COPD was diagnosed taking into account medical history, objective examination, peak flow measurements, spirography with a bronchodilator test (spirograph "FUKUDA", Japan), results of Modified Medical Research Council (mMRC) and COPD Assessment Test (CAT) tests, X-ray, laboratory analyses, and according to GOLD recommendations [10] (forced expiratory volume in the first second / forced vital capacity (FEV1/FVC) < 0.70) (Table 1). All patients with COPD received basic therapy with long-acting $m$ cholinolytics, patients with severe COPD also received long-acting $m$-cholinolytics combined with a fixed combination of long-acting $\beta 2$-agonists and inhaled glucocorticosteroids [10].

\section{Determination of Th immune response direction}

Serum cytokine levels (tumor necrosis factor- $\alpha$ (TNF- $\alpha$ ), interferon- $\gamma$ (IFN- $\gamma)$, IL-4, IL-6, IL-10, IL-17A) were investigated using flow cytometry (cytometer "BD FACSCantoll"; test system: "BD", USA). Data processing was performed using "BD FCAP Array 3.0" software (USA). The serum levels of transforming growth factor- $\beta 1$ (TGF- $\beta 1$ ) and IL-21 were assessed by ELISA ("Genzyme diagnostics", USA). The direction of immune response in COPD patients was determined depending on the prevalence of cytokines playing a crucial role in the formation of certain Th cells 
type (Th1, Th17). In accordance with the detected Th-associated cytokine profile, patients with COPD were divided into the following subgroups: subgroups 1 a (patients with mild COPD and Th1-associated cytokine profile), 2a (patients with moderate COPD and Th1-associated cytokine profile), 3a (patients with severe COPD and Th1-associated cytokine profile) and subgroup $1 \mathrm{~b}$ (patients with mild COPD and Th17-associated cytokine profile), 2b (patients with moderate COPD and Th71-associated cytokine profile), $3 \mathrm{~b}$ (patients with severe COPD and Th17-associated cytokine profile).

\section{Determination of IL-6R expression level in blood Th cells}

The percentage of T-helper cells $\left(\mathrm{CD}^{+}\right)$expressing membrane IL-6R $\left(\mathrm{CD} 126^{+}\right)$in peripheral blood was determined by flow cytometry (cytometer "BD FACSCantoll", USA) using labeled monoclonal antibodies - CD45 (APC-H7), CD3 (FITC), CD4 (PE-Cy7), CD126 (APC) ("BD", USA).

\section{Statistical analysis}

Statistical data processing was performed using "Statistica 6.0" software. Kolmogorov - Smirnov test was used to check normality of the distribution for the studied quantitative indicators. The results of statistical processing for data with non-normal distribution were presented as median, upper and lower quartiles. Bonferroni correction was used to solve the problem of multiple comparisons. The non-parametric Mann-Whitney U-test was applied to identify significant differences of compared indicators. According to Bonferroni correction, differences were considered significant at $p<0.00714, p<0.00143, p<0.00014$.

\section{Results}

\section{The features of cytokine profile in COPD of different severity}

The assessment of circulating cytokines levels has shown that inflammation in COPD is characterized by cytokine profile associated with Th1 or Th17 immunoregulatory pathway (Table 2, $3)$. As the pathology progressed, the increase in number of patients with Th17-associated cytokine profile was observed.

Subgroup $1 \mathrm{a}$ included $67 \%$ of patients of group 1 . An increase in the concentrations of IFN- $\gamma$ and TNF- $\alpha$, a decrease in IL-4 $(p<0.00714)$ level in the blood were found in this subgroup compared to the control group. Subgroup $2 a$ included $46 \%$ of patients of group 2. There was a decrease in the serum level of IL-4 and an increase in the production of IL-10, TNF- $\alpha$ and IFN- $\gamma$ $(p<0.00714)$ in these patients compared to the control values. Subgroup 3a consisted of $25 \%$ of patients of group 3 . High levels of IL-6, TNF- $\alpha$, IFN- $\gamma$ and TGF- $\beta 1$ against the low level of IL-21 $(p<0.00714)$ were found in this subgroup (Table 2$)$.

Subgroup $1 \mathrm{~b}$ consisted of $33 \%$ of patients of group 1 . This subgroup was characterized by elevated levels of IL-6, IL-17A, IL10, IL-21, TNF- $\alpha$, TGF- $\beta 1$ and decreased IL-4 $(p<0.00714)$ level in the blood compared to the control group. Subgroup $2 \mathrm{~b}$ included $54 \%$ of patients of group 2. Th-associated cytokine profile of patients of this subgroup was described by high concentrations of IL-6, IL-10, IL-17A, IL-21 and TGF- $\beta 1$ ( $p<0.00714)$ in the blood. Most patients of group $3(75 \%)$ were included in subgroup $3 \mathrm{~b}$. The maximal concentrations of IL-6, IL-10, IL-17A, IL-21, TNF- $\alpha$ and TGF- $\beta 1(p<0.00714)$ were identified in this subgroup (Table 3$)$.

\section{Dynamics of IL-6R expression level in blood T-helpers in COPD patients}

The investigation of IL-6R expression in $\mathrm{CD}^{+}$population of peripheral blood of COPD patients showed different dynamics of the parameter values depending on prevailing Th-associated cytokine profile (Table 2,3 ).

The patients of subgroup 1a showed tendency for an increase (by $37 \%$ ) in the relative number of $\mathrm{CD} 4^{+} \mathrm{CD} 126^{+}$cells compared to the control group. A statistically significant decrease in the number of $\mathrm{CD} 126^{+}$T-helpers by $14 \%(p<0.00714)$ compared to healthy subjects was observed in subgroup 2 a. In patients of subgroup $3 a$ the levels of $\mathrm{CD} 4^{+} \mathrm{CD} 126^{+}$cells have exceeded the control value by 111\% ( $p<0.00014$ ) (Table 2).

An increase in the number of Th cells expressing IL-6R by $47 \%$ $(p<0.00714)$ relative to the control group was found in patients of subgroup $1 \mathrm{~b}$. The percentage of Th cells with $\mathrm{CD}_{126^{+}}$was increased by $117 \%(p<0.00143)$ in subgroup $2 b$, compared to healthy volunteers. The patients of subgroup $3 \mathrm{~b}$ exhibited the increased levels of $\mathrm{CD}^{+} \mathrm{CD} 126^{+}$cells (by $286 \% \quad(p<0.00143)$ ) compared to the control group (Table 3 ).

\section{Discussion}

Th cells play a key role in systemic inflammatory process during COPD. Various Th subpopulations are able to control the development of immune response by activating corresponding effector cells. In turn, the differentiation of Th subpopulations depends on cytokine microenvironment [1]. In our study, we defined subgroups of COPD patients with Th1- and Th17associated cytokine profiles according to the ratios of serum cytokine levels. The results are consistent with modern literature data that suggest an increase in the number of Th1 and Th17 cells in the blood of COPD patients [2, 3].

Considering cytokine balance in COPD with different severity, it has been found that cytokine profile corresponding to the formation of Th1 immune response prevails in patients with mild COPD. Macrophages and T-killer cells are the main effector cells for this immune response type. This immunoregulatory pathway is accompanied by an increase in the levels of pro-inflammatory cytokines IFN- $\gamma$ and TNF- $\alpha$, and a decrease in IL- 4 concentration. This indicates the initiation of inflammatory process and the disruption of anti-inflammatory component in mild COPD. Increased levels of IFN- $\gamma$ and TNF- $\alpha$ in the serum of COPD patients have been reported in other studies [11]. The combination of IL-4 deficiency, a key factor for differentiation of Th2 cells, and the high levels of IFN- $\gamma$, which is essential for Th2 differentiation, indicates the activation of naive T-lymphocytes differentiation into Th1 cells that are responsible for the formation of the appropriate type of immune response.

Th17-associated cytokine profile begins to prevail as the disease progresses (moderate and severe COPD). It may be explained by Th17 cells forming their own immunoregulatory pathway if an effective elimination of the pathogen does not occur during the Th1-dependent immune response at the initial stage of COPD. According to the obtained results, IL-6, IL-17A, IL-21, and TGF- $\beta 1$ make the greatest contribution to the development of this immune response type in COPD. At the same time, the enhanced synthesis of anti-inflammatory IL-10, which is aimed at restoring the cytokine balance, has been observed. High levels of IL-6, IL-21 and TGF- $\beta 1$ in COPD patients indicate the formation of a specific cytokine pattern leading to the differentiation of Th17 cells. These 
cells actively produce IL-17, which contributes to the activation of pulmonary fibroblasts, epithelial cells and smooth muscle cells of the bronchi. After activation these cells express cytokines (IL-6, IL8 and granulocyte colony-stimulating factor (G-CSF)), causing the infiltration of respiratory tract by neutrophils and an increase in their proliferation. Performing their effector functions, neutrophils die and release a large number of proteolytic enzymes (neutrophilic elastase, cathepsin G, protease 3 and matrix metalloproteinases), which damage the lung tissue and enhance the chemotaxis of immune cells to the inflammatory focus $[2,3]$. Thus, the initiation of the Th17 immunoregulatory pathway leads to the prolongation of innate immune response and, consequently, to the aggravation of inflammation in COPD.

Several studies have emphasized that an increased systemic level of IL- 6 is a hallmark of COPD, also correlating with the degree of inflammation in lower respiratory tract and a decrease in pulmonary function $[7,11,12]$. In our study we showed that COPD progression in patients with Th17-associated cytokine profile is accompanied by an increase in IL- 6 levels. These data indicate the important role of this cytokine in the formation of Th17 immune response in COPD. Although the absence of differences in IL-6 blood levels between patients with mild COPD and patients with severe COPD has been described in a systematic review wrote by Wei et al. [13], our results pointed out a significant difference in IL6 levels between these groups of patients. We assume that this difference is due to a change in the direction of Th immune response. An important property of IL-6 is its ability to influence the differentiation of Th-lymphocytes which determine the development direction of adaptive immune response [14].

According to modern studies, the implementation of IL-6 properties depends on the type of cell signaling [9]. The IL-6 receptor complex consists of IL-6R subunit, which is responsible for cytokine binding, and gp130 subunit, which provides JAK / STAT (janus kinase / signal transducer and activator of transcription protein) or MAPK (mitogen-activated protein kinase) signaling cascades. There are 2 main types of IL- 6 signaling: classical signaling (via IL-6R membrane form) and trans-signaling (via IL-6R soluble form (sIL-6R)), which cause various pathophysiological and clinical effects of this multifunctional cytokine $[14,15]$. Despite the considerable interest of researchers in IL-6, the exact mechanisms of IL-6 impact on Th immune response are still unknown. According to the hypothesis of Lissilaa et al., classical IL-6 signaling is involved in systemic reactions of T-cell response, while IL- 6 trans-signaling controls the local effects of IL- 6 in focus of inflammation. This assumption was based on the study of an experimental model of rheumatoid arthritis [16]. Jones et al. have reported that classic IL-6 signaling in Th cells is essential to control effector functions of these cells, while trans-signaling maintains the cytokine profile of Th cell infiltrate in inflammatory focus [17]. Therefore, the activation status of Th cells is associated with type of IL- 6 signaling. It should be noted that activated $\mathrm{CD}^{+}$cells in inflammatory tissue are able to produce sIL-6R by limited proteolysis that serves as the main mechanism for generating soluble form of IL- 6 receptor $[15,18]$. No similar studies have been conducted for COPD.

Analyzing current data on the IL- 6 role in COPD pathogenesis, we can conclude that more attention is paid to the study of transsignaling as opposed to classical signaling. Studies have found an increase in sIL-6R levels in the blood and sputum of COPD patients [18] that indicates its important pathological role in this disease. It has reported that IL- 6 provides for the recruitment of innate immunity cells in inflammatory focus in COPD by activating chemokine synthesis in endothelial cells via trans-signaling [9]. Le et. al. have demonstrated in an experimental study that sIL-6R subunit inhibition leads to a reduction in the number of fibroblasts in lung tissue and, as a consequence, a decrease in the intensity of extracellular matrix protein synthesis and a decrease in lung fibrosis [19]. Robinson et al. have showed that IL-6 trans-signaling in human smooth muscle cells of the respiratory tract induces the expression of genes involved in airway remodeling in these cells [20].

In our earlier studies, it has been shown that IL-6R expression on the surface of circulating Th cells increases at last stages of COPD [21], thus indicating the importance of the activation of classical IL-6 signaling in these cells for the inflammatory process development during disease. In this study, we have found that severe COPD development accompanied by the formation of Th17associated cytokine profile is characterized by increase in the IL- 6 blood levels and the number of Th cells expressing IL-6R. According to these data, it can be assumed that classic IL-6 signaling in $\mathrm{CD}^{+}{ }^{+}$cells plays an important role in the development of Th17 pathway. These data are consistent with the results of the study by Chen et al., in which researchers have established the correlation between the suppression of Th17 immune response, which was observed in patients with tuberculosis, and a decrease in IL-6R expression in CD4+ cells [22]. It is known that IL-6 serves as a key factor in the differentiation of Th17 cells, exhibits suppressor activity against Th1 and Treg subpopulations, and is also able to inhibit cell apoptosis by inducing anti-apoptotic regulators [2, 3]. Taking into account these data, we suggest that classical IL-6 signaling contributes to the development of Th17 immune response by inhibiting Th cells apoptosis and activating the differentiation of Th17 lymphocytes. In addition, we have found that COPD patients with Th1-associated cytokine profile have a significant increase in IL-6 synthesis and IL-6R expression in CD4 ${ }^{+}$ blood cells only at the severe stage of disease. This may be due to the repolarization of Th1 phenotype towards Th17 direction as the pathology progresses.

Thus, the mechanisms of cytokine regulation disorders in COPD and their impact on the formation of Th immune response require further study. The understanding of these mechanisms will allow developing new approaches to anti-cytokine therapy, diagnosis and prognosis of inflammatory response in COPD patients in the future.

\section{Conclusion}

Inflammation in stable COPD is characterized by the formation of Th1- or Th17-associated cytokine profile. Th1-associated cytokine profile develops at the initial stage of COPD; the Th17associated cytokine profile, leading to chronic inflammation, begins to prevail in severe COPD. The increase in the expression levels of IL-6R, which mediates the classical IL- 6 signaling, in circulating $\mathrm{CD}^{+}{ }^{+}$cells serves as the mechanism for enhancing Th17mediated response in severe COPD. The expression level of IL-6R in $\mathrm{CD}^{+}$cells can be used as a diagnostic criterion for the assessment of the intensity of inflammation in COPD patients with Th17-associated cytokine profile.

\section{Ethical approval}

All test subjects gave an informed consent for inclusion prior to their participation in the study. The study was conducted in accordance with the 
Declaration of Helsinki, and the protocol was approved by the Ethics Committee of the Vladivostok Branch of Federal State Budgetary Science Institution "Far Eastern Scientific Center of Physiology and Pathology of Respiration" - Institute of Medical Climatology and Rehabilitative Treatment (identification code: 18/12-17, approval date: December 15, 2017).

\section{Conflict of interest}

The authors declare that they have no conflict of interest.

\section{Acknowledgements}

The reported study was funded by RFBR, project number 19-31590029.

\section{References}

1. Barnes PJ. Inflammatory mechanisms in patients with chronic obstructive pulmonary disease. J Allergy Clin Immunol 2016; 138(1): 16-27. https://doi.org/10.1016/j.jaci.2016.05.011.

2. Wang $H$, Ying $H$, Wang $S, G u X$, Weng $Y$, Peng $W$, et al. Imbalance of peripheral blood Th17 and Treg responses in patients with chronic obstructive pulmonary disease. Clin Respir J 2015; 9(3): 330-341. https://doi.org/10.1111/crj.12147.

3. Cosmi L, Liotta F, Annunziato F. Th17 regulating lower airway disease. Curr Opin Allergy Clin Immunol 2016; 16(1): 1-6. https://doi.org/10.1097/aci.0000000000000227.

4. Matera MG, Page C, Rogliani P, Calzetta L, Cazzola M. Therapeutic monoclonal antibodies for the treatment of chronic obstructive pulmonary disease. Drugs 2016; 76(13): 1257-1270. https://doi.org/10.1007/s40265-016-0625-9.

5. Zhu J, Yamane H, Paul WE. Differentiation of effector CD4 T cell populations. Annu Rev Immunol 2009; 28: 445-489. https://doi.org/10.1146/annurev-immunol-030409-101212.

6. Unver N, McAllister F. IL-6 family cytokines: key inflammatory mediators as biomarkers and potential therapeutic targets. Cytokine Growth Factor Rev 2018; 41: 10-17. https://doi.org/10.1016/j.cytogfr.2018.04.004.

7. Singh S, Verma SK, Kumar S, Ahmad MK, Nischal A, Singh SK, et al. Correlation of severity of chronic obstructive pulmonary disease with potential biomarkers. Immunol Lett 2018; 196: 1-10. https://doi.org/10.1016/j.imlet.2018.01.004.

8. Celli BR, Locantore N, Yates J, Tal-Singer R, Miller BE, Bakke P, et al. Inflammatory biomarkers improve clinical prediction of mortality in chronic obstructive pulmonary disease. Am J Respir Crit Care Med 2012; 185(10): 1065-1072. https://doi.org/10.1164/rccm.2011101792 oc.

9. Vitkina $\mathrm{TI}$, Sidletskaya KA. The role of interleukin-6 signaling in development of systemic inflammation in chronic obstructive pulmonary disease. Bulletin Physiology and Pathology of Respiration 2018; (69): 96-106. Russian. https://doi.org/10.12737/article 5b9858ead1b5e3.93619630.

10. Global Initiative for Chronic Obstructive Lung Disease, Pocket Guide to COPD Diagnosis, Management, and Prevention. A Guide for Health Care Professionals. Global Initiative for Chronic Obstructive Lung Disease Inc, 2017; 37 p. https://goldcopd.org/wpcontent/uploads/2016/12/wms-GOLD-2017-Pocket-Guide.pdf.

11. Bradford E, Jacobson S, Varasteh J, Comellas AP, Woodruff P, O'Neal $\mathrm{W}$, et al. The value of blood cytokines and chemokines in assessing COPD. Respir Res 2017; 18(1): 180. https://doi.org/10.1186/s12931017-0662-2.

12. Vitkina TI, Denisenko YuK, Davydova KA. The changes in the profile of cytokines in progressing chronic obstructive pulmonary disease. Int Res J 2016; (7 pt 3): 6-8. https://doi.org/10.18454/IRJ.2016.49.024.

13. Wei J, Xiong XF, Lin YH, Zheng BX, Cheng DY. Association between serum interleukin- 6 concentrations and chronic obstructive pulmonary disease: a systematic review and meta-analysis. PeerJ 2015; 3: e1199. https://doi.org/10.7717/peerj.1199.

14. Hunter CA, Jones SA. IL-6 as a keystone cytokine in health and disease. Nat Immunol 2015; 16(5): 448-457. https://doi.org/10.1038/ni.3153.

15. Garbers C, Aparicio-Siegmund S, Rose-John S. The IL-6/gp130/STAT3 signaling axis: recent advances towards specific inhibition Curr Opin Immunol 2015; 34: 75-82. https://doi.org/10.1016/j.coi.2015.02.008.

16. Lissilaa R, Buatois V, Magistrelli G, Williams AS, Jones GW, Herren S, et al. Although IL-6 trans-signaling is sufficient to drive local immune responses, classical IL-6 signaling is obligate for the induction of T cellmediated autoimmunity. J Immunol 2010; 185: 5512-5521. https://doi.org/10.4049/jimmunol.1002015.

17. Jones GW, McLoughlin RM, Hammond VJ, Parker CR, Williams JD, Malhotra $\mathrm{R}$, et al. Loss of $\mathrm{CD} 4+\mathrm{T}$ cell IL-6R expression during inflammation underlines a role for IL- 6 trans signaling in the local maintenance of Th17 cells. J Immunol 2010; 184(4): 2130-2139. https://doi.org/10.4049/jimmunol.0901528.

18. Ravi AK, Khurana S, Lemon J, Plumb J, Booth G, Healy L, et al. Increased levels of soluble interleukin-6 receptor and CCL3 in COPD sputum. Respir Res 2014; 15(1): 103. https://doi.org/10.1186/s12931-0140103-4.

19. Le TT, Karmouty-Quintana H, Melicoff E, Le TT, Weng T, Chen NY, et al. Blockade of IL-6 trans signaling attenuates pulmonary fibrosis. J Immunol 2014; 193(7): 3755-3768. https://doi.org/10.4049/jimmunol.1302470.

20. Robinson MB, Deshpande DA, Chou J, Cui W, Smith S, Langefeld C, et al. IL-6 trans-signaling increases expression of airways disease genes in airway smooth muscle. Am J Physiol Lung Cell Mol Physiol 2015; 309(2): L129-L138. https://doi.org/10.1152/ajplung.00288.2014.

21. Vitkina TI, Denisenko YuK, Sidletskaya KA. Changes in the surface IL-6 receptor expression of distinct immune cells in progression of chronic obstructive pulmonary disease. Medical Immunology (Russia) 2017 19(2): 191-196. Russian. https://doi.org/10.15789/1563-0625-2017-2 191-196.

22. Chen $X$, Zhang $M$, Liao $M$, Graner $M W$, Wu $C$, Yang $Q$, et al. Reduced Th17 response in patients with tuberculosis correlates with IL-6R expression on CD4+ T Cells. Am J Respir Crit Care Med 2010; 181(7) 734-742. https://doi.org/10.1164/rccm.200909-1463oc.

\section{Authors:}

Tatyana I. Vitkina - MD, PhD (Biology), Professor of Russian Academy of Sciences, Leading Researcher, Laboratory of Biomedical Research, Vladivostok Branch of Far Eastern Scientific Center of Physiology and Pathology of Respiration - Institute of Medical Climatology and Rehabilitative Treatment, Vladivostok, Russia. http://orcid.org/0000-00021009-9011.

Karolina A. Sidletskaya - PhD student, Junior Researcher, Laboratory of Biomedical Research, Vladivostok Branch of Far Eastern Scientific Center of Physiology and Pathology of Respiration - Institute of Medical Climatology and Rehabilitative Treatment, Vladivostok, Russia. https://orcid.org/0000$\underline{0003-4201-9167}$ 